\title{
Multiple wavelength InGaAs quantum dot lasers using selective area epitaxy
}

\author{
S. Mokkapati, ${ }^{\text {a) }}$ H. H. Tan, and C. Jagadish \\ Department of Electronic Materials Engineering, Research School of Physical Sciences and Engineering, \\ The Australian National University, Canberra, Australian Capital Territory 0200, Australia
}

(Received 20 February 2007; accepted 28 March 2007; published online 24 April 2007)

\begin{abstract}
The authors demonstrate multiple wavelength lasers fabricated from InGaAs quantum dots. Selective area epitaxy is used to grow the active region, consisting of five layer stack of InGaAs quantum dots with different band gap energies in selected regions of the substrate, for fabrication of the lasers. The mechanism responsible for engineering of the band gap of quantum dots is discussed. The performance of the selectively grown lasers is compared to the lasers fabricated from structures grown in a standard, nonselective area growth process. (C) 2007 American Institute of Physics.
\end{abstract} [DOI: $10.1063 / 1.2731729$ ]

Following the demonstration of quantum dot (QD) lasers with low threshold currents $^{1-3}$ and good temperature stabilities, ${ }^{3-5}$ there has been a lot of interest in engineering the band gap of QDs. Controlling the area of nucleation of QDs (Ref. 6) and the ability to engineer their band gap are essential for integration of QD-based optoelectronic devices. Integration of several optoelectronic devices on a single chip offers advantages of compact size, better performance, and low optical losses over discrete devices coupled through fibers. We have shown earlier that selective area epitaxy (SAE) can be used to tune the band gap of QDs formed in the Stranski-Krastanow growth mode. ${ }^{7}$ SAE has been widely used for integration of quantum well (QW) optoelectronic devices $^{8-11}$ and recently, integration of a QD laser with a passive (QW) waveguide has been demonstrated. ${ }^{12}$ In this letter, we report on InGaAs QD lasers, emitting at different wavelengths, fabricated on the same substrate. QDs with different band gap energies are nucleated in different parts of the same substrate in a single growth step using SAE. Control of local growth rate enhancement and In incorporation into epitaxially grown layers using a dielectric mask $\left(\mathrm{SiO}_{2}\right.$ in this work) is used to engineer the band gap of QDs.

The structures studied in this work were grown by lowpressure, horizontal flow metal-organic chemical vapor deposition (MOCVD) system. The precursor sources used were trimethylindium, trimethylgallium, and $\mathrm{AsH}_{3}$ with $\mathrm{H}_{2}$ as the carrier gas. Silane and $\mathrm{CCl}_{4}$ were used as $n$ - and $p$-type dopant sources, respectively. A three-step growth process developed to avoid the problems associated with selective area growth of $\mathrm{Al}$ containing compounds ${ }^{13}$ was used to grow the device structures. The samples were patterned with $100 \mathrm{~nm}$ of $\mathrm{SiO}_{2}$ for growing the active region consisting of five layers of InGaAs QDs. Figure 1(a) shows the schematic of the mask pattern used in this work. It consists of pairs of $\mathrm{SiO}_{2}$ stripes, separated from each other by $50 \mu \mathrm{m}$. The widths of the stripes are 5, 10, 15, and $20 \mu \mathrm{m}$. Figure 1(b) shows the cross-section schematic of the device structures. $4 \mu \mathrm{m}$ wide ridge waveguide lasers, centered on the openings between the $\mathrm{SiO}_{2}$ stripes, were fabricated using the standard laser processing steps. ${ }^{14}$ Lasers were also fabricated from device structures grown in a standard single growth step

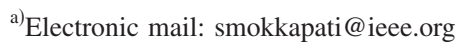

(without mask) for comparison. All the devices were tested as cleaved (no facet coatings applied) at room temperature in pulsed mode.

During the growth of the active region, no deposition takes place on the $\mathrm{SiO}_{2}$ stripes. Surface migration and gas phase diffusion of adatoms from masked regions to the unmasked areas cause enhanced deposition rates in these regions. The growth rate enhancement can be locally controlled by controlling the dimensions of the mask used for patterning the substrate. ${ }^{7}$ The growth conditions were optimized so that the thickness of InGaAs deposited on unpatterned substrates is just below the critical thickness for QD nucleation, so that no QDs are formed on unpatterned regions. But due to the local growth rate enhancement, QDs are formed between the stripes. Thus the size, composition (which determines the band gap), and density of the QDs formed (which determines the amount of gain available for the lasers) are controlled by the stripe width.

For a stripe width of $5 \mu \mathrm{m}$, the growth rate enhancement is minimal and QDs of low density are formed. The QD density is not sufficient for providing enough gain for ground state lasing. So devices fabricated from these QDs lase from QD excited states. Electroluminescence (EL) spectra for a representative device are shown in Fig. 2(a). The lumines-

(a)
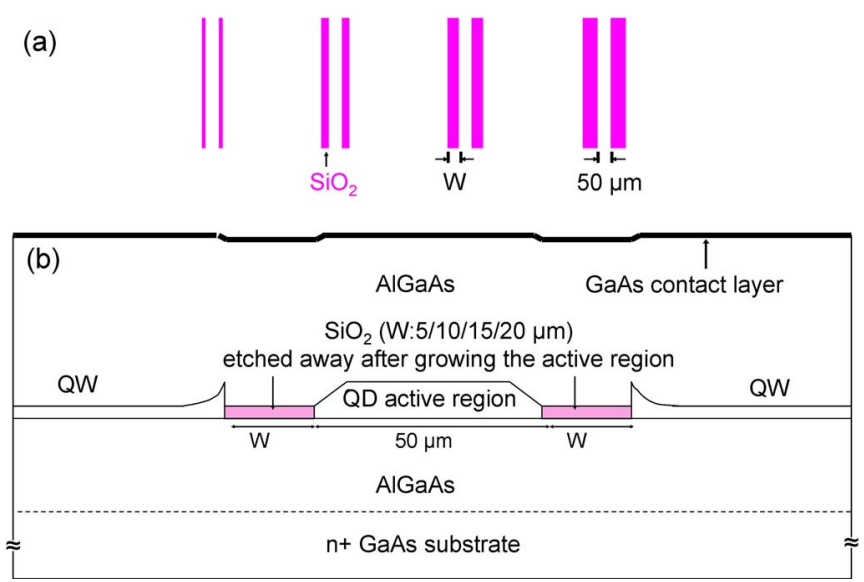

FIG. 1. (Color online) Schematic showing (a) the dimensions of the mask used for growing the active region for devices studied in this work and (b) cross section of the completed device structure. 


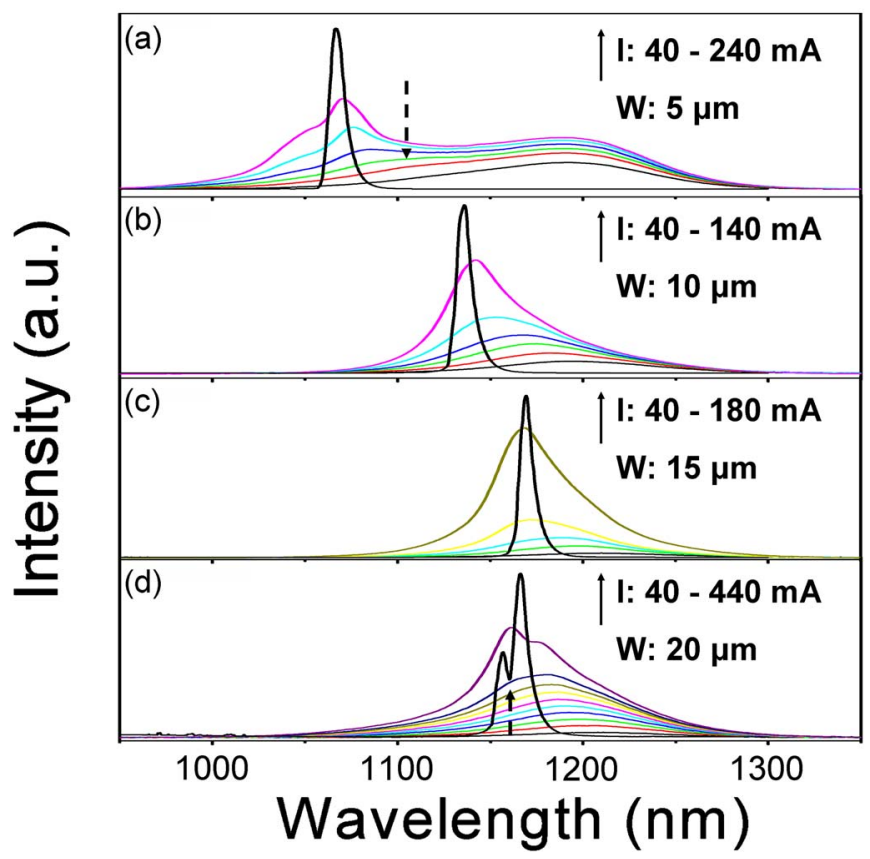

FIG. 2. (Color online) Electroluminescence and lasing spectra of $3 \mathrm{~mm}$ long lasers fabricated from QDs grown between pairs of dielectric stripes of widths (W) (a) $5 \mu \mathrm{m}$, (b) $10 \mu \mathrm{m}$, (c) $15 \mu \mathrm{m}$, and (d) $20 \mu \mathrm{m}$. The separation between the $\mathrm{SiO}_{2}$ stripes is $50 \mu \mathrm{m}$. Luminescence peaks from QD excited states are indicated by dashed arrows in (a) and (d).

cence from the QD ground state peaks at $\sim 1190 \mathrm{~nm}$ at an injection current of $40 \mathrm{~mA}$ and luminescence from higher lying energy states appears at $\sim 1115 \mathrm{~nm}$. As more current is injected into the device, the luminescence peak from the excited states blueshifts, until the device starts to lase at $1070 \mathrm{~nm}$.

When the stripe width is increased to $10 \mu \mathrm{m}$, the growth rate enhancement is sufficient to form a high density of QDs for the same deposition time. The increase in the QD density now provides enough ground state gain to overcome the cavity losses and internal optical losses, and the lasers fabricated from these QDs lase from the ground state. Figure 2(b) shows EL spectra for a $3 \mathrm{~mm}$ long device fabricated from QDs grown between $10 \mu \mathrm{m}$ wide $\mathrm{SiO}_{2}$ stripes. The luminescence from the QDs peaks at $\sim 1195 \mathrm{~nm}$ for an input current of $40 \mathrm{~mA}$. The luminescence peak blueshifts by $60 \mathrm{~nm}$ with increasing injection current, until the device starts to lase at $\sim 1135 \mathrm{~nm}$.

By increasing the stripe width to $15 \mu \mathrm{m}$, the growth rate enhancement is sufficient to form QDs with smaller band gap energies. The variation in QD band gap is due to variations in dot size and composition, which in turn occurs because of differential incorporation of InAs and GaAs into the dots. ${ }^{7}$ Figure 2(c) shows the EL spectra for a $3 \mathrm{~mm}$ long laser fabricated from QDs grown between $15 \mu \mathrm{m}$ wide $\mathrm{SiO}_{2}$ stripes. The luminescence from these QDs peaks at $\sim 1210 \mathrm{~nm}$ at an injection current of $40 \mathrm{~mA}$. As the device is pumped harder, the luminescence peak blueshifts by $40 \mathrm{~nm}$ and the device starts to lase at $1170 \mathrm{~nm}$. The EL spectra of the device do not show any luminescence from higher lying excited states.

By increasing the stripe width to $20 \mu \mathrm{m}$, there is no further variation in the QD band gap energy. The increase in the total amount of material deposited due to the enhanced growth rate increases the strain energy, which promotes forDownloaded 24 Feb 2008 to 150.203 .178 .41 . Redistribution subject
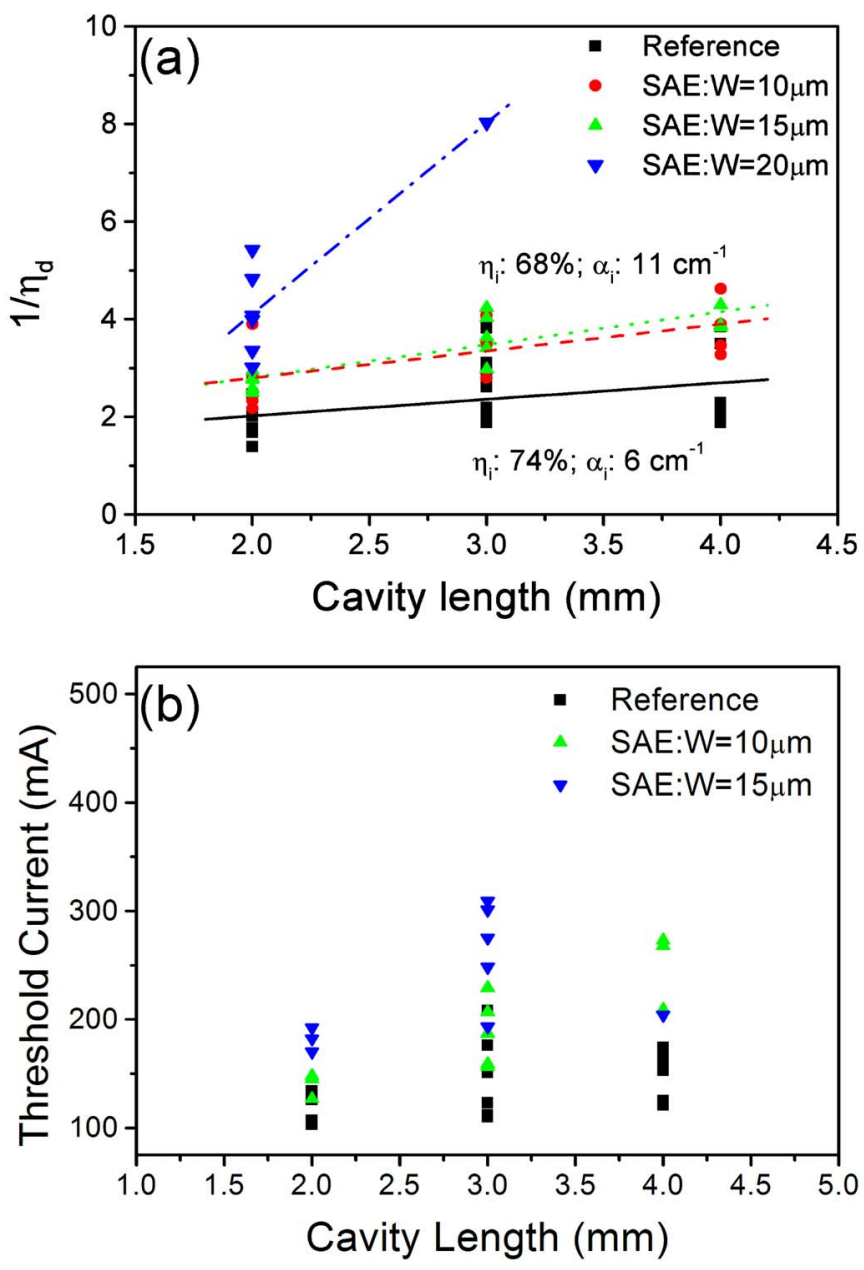

FIG. 3. (Color online) (a) Inverse differential efficiency vs cavity length plot for lasers fabricated from QDs grown between $\mathrm{SiO}_{2}$ stripes of widths $(W)$ 10, 15, and $20 \mu \mathrm{m}$. (b) Threshold currents of lasers fabricated from QDs grown between $\mathrm{SiO}_{2}$ stripes of widths $(W) 10$ and $15 \mu \mathrm{m}$. Inverse differential efficiency and threshold currents for a reference device, fabricated from QDs grown on unpatterned substrates, are also shown. The separation between the $\mathrm{SiO} 2$ stripes is $50 \mu \mathrm{m}$.

mation of defects. Once defects appear in the QDs, any additional material deposited is preferentially accumulated at these defects and does not contribute to varying the average size of the optically active QDs. So devices fabricated from QDs grown between $20 \mu \mathrm{m}$ wide $\mathrm{SiO}_{2}$ stripes do not lase at longer wavelengths than the devices fabricated from QDs grown between $15 \mu \mathrm{m}$ wide stripes. However, due to the increased defect density in the active region, these devices exhibit very high internal losses [Fig. 3(a)]. For cavity lengths of $3 \mathrm{~mm}$, the devices fabricated from dots grown between $20 \mu \mathrm{m}$ wide stripes lase at slightly shorter wavelengths than the ones fabricated from QDs grown between $15 \mu \mathrm{m}$ wide stripes, as these devices have to be pumped harder in order to overcome increased losses, which makes them lase from excited states. Figure 2(d) shows EL spectra for a typical device. The luminescence from the ground state of QDs peaks at $1210 \mathrm{~nm}$ at an injection current of $40 \mathrm{~mA}$. Luminescence from excited states starts to appear at $\sim 1164 \mathrm{~nm}$ for an input current of $360 \mathrm{~mA}$ and the device finally lases at $1160 \mathrm{~nm}$.

We now present results on the performance of devices fabricated from QDs grown between $\mathrm{SiO}_{2}$ stripes of widths 10 and $15 \mu \mathrm{m}$. Figure 3 (a) compares the inverse differential
to AIP license or copyright; see http://apl.aip.org/apl/copyright.jsp 
efficiency versus cavity length plots for these two devices with that of lasers fabricated from QDs grown on unpatterned substrates. The two selectively grown devices have similar internal optical losses of $\sim 11 \mathrm{~cm}^{-1}$, while devices fabricated from QDs grown on unpatterned substrates have losses of $6 \mathrm{~cm}^{-1}$. In Fig. 3(b) we compare the threshold currents of the two SAE grown devices with those of devices fabricated from QDs grown on unpatterned substrates for various device lengths. The two selectively grown devices have similar threshold currents, which are slightly higher than those for devices of the same length fabricated from laser structures grown in a single growth step. A slight increase in the internal losses and threshold currents could be due to the quality of regrowth interfaces and midgrowth processing steps.

The EL spectra shown in Figs. 2(b) and 2(c) show different amounts of blueshifts before lasing. The amount of blueshift in the EL spectra of a device depends on dot density (lower dot density requires harder pumping, leading to a population of higher lying energy states which causes more shift), built-in electric dipole moment (which arises in the case of MOCVD grown QDs due to In segregation ${ }^{15,16}$ ), and QD size distribution. ${ }^{17}$ Since we have already shown that the two SAE grown devices have similar losses and threshold currents, the contribution of dot density variations to varying shifts in the EL spectra can be ruled out. It has been shown that band gap tuning of QDs is due to the variation in their size and composition. ${ }^{7}$ These variations affect the process of In segregation, which in turn affects the built-in electric dipole moment. We attribute different amounts of blueshifts in the EL spectra depicted in Figs. 2(b) and 2(c) to variations in built-in electric dipole moment and dot size distributions. These observations are consistent with data showing smaller blueshift in the EL spectra of In rich QDs grown using MOCVD. ${ }^{17,18}$

Although the simplicity of postgrowth processing techniques makes them attractive for integration of optoelectronic devices, SAE clearly has its advantages over these techniques. The postgrowth processing techniques involve heat treatment of the device structures, which blueshifts the emission wavelength of the QDs. It also leads to diffusion of dopants from the cladding and contact layers and reduces the confinement potential and thus the carrier capture efficiency of QDs in the active region of the devices, which decreases the efficiency of these devices; whereas SAE devices are comparable to normal, as grown devices. The longer emission wavelengths for integrated devices fabricated using In(Ga)As QDs that are reported in this work cannot be obtained using postgrowth processing techniques.

To conclude, we have demonstrated ground state lasing from multiple wavelength $(\Delta \lambda \sim 35 \mathrm{~nm})$ InGaAs QD lasers.
Control of local growth rate enhancement by varying the local mask dimensions during selective area MOCVD is used to engineer the band gap of QDs in the active region of the lasers. The shift in the lasing wavelength of devices fabricated using these QDs is increased further by variations in built-in electric dipole moment and variations in dot size distribution. These devices have very similar internal efficiencies, internal optical losses, and threshold currents and are comparable in performance to devices fabricated from QDs grown on unpatterned substrates. However, if the mask dimensions are not optimized, either too little or too much material may be deposited during the growth of the active region, leading to lasing from QD excited states.

Thanks are due to Michael Aggett for technical assistance. The Australian Research Council is gratefully acknowledged for financial support.

${ }^{1}$ G. T. Liu, H. Li, A. Stintz, T. C. Mewell, A. L. Gray, P. M. Varangis, K. J. Malloy, and L. F. Lester, IEEE J. Quantum Electron. 36, 1272 (2000).

${ }^{2}$ I. R. Sellers, H. Y. Liu, K. M. Groom, D. T. Childs, D. Robbins, T. J. Badcock, M. Hopkinson, D. J. Mowbray, and M. S. Skolnick, Electron. Lett. 40, 1412 (2004).

${ }^{3}$ A. R. Kovsh, N. A. Maleev, A. E. Zhukov, S. S. Mikhrin, A. P. Vasil'ev, Yu. M. Shernyakov, M. V. Maximov, D. A. Livshits, V. M. Ustinov, Zh. I. Alferov, N. N. Ledentsov, and D. Bimberg, Electron. Lett. 38, 1104 (2002).

${ }^{4}$ O. B. Shchekin and D. G. Deppe, Appl. Phys. Lett. 80, 3277 (2002).

${ }^{5}$ S. Fathpour, Z. Mi, P. Bhattacharya, A. R. Kovsh, S. S. Mikhrin, I. L. Krestnikov, A. V. Kozhukhov, and N. N. Ledentsov, Appl. Phys. Lett. 85, 5164 (2004).

${ }^{6}$ J. Tatebayashi, M. Nishioka, T. Someya, and Y. Arakawa, Appl. Phys. Lett. 77, 3382 (2000).

${ }^{7}$ S. Mokkapati, P. Lever, H. H. Tan, C. Jagadish, K. E. McBean, and M. R. Phillips, Appl. Phys. Lett. 86, 113102 (2005).

${ }^{8}$ Kostadin Djordjev, Sang-Jun Choi, Won-Jin Choi, Seung-June Choi, In Kim, and P. D. Dapkus, IEEE Photonics Technol. Lett. 14, 603 (2002).

${ }^{9}$ R. M. Lammert, G. M. Smith, D. V. Forbes, M. L. Osowski, and J. J. Coleman, Electron. Lett. 31, 1070 (1995).

${ }^{10}$ R. M. Lammert, G. M. Smith, J. S. Hughes, M. L. Osowski, A. M. Jones, and J. J. Coleman, IEEE Photonics Technol. Lett. 8, 797 (1996).

${ }^{11}$ R. M. Lammert, S. D. Roh, J. S. Hughes, M. L. Osowski, and J. J. Coleman, IEEE Photonics Technol. Lett. 9, 566 (1997).

${ }^{12}$ S. Mokkapati, H. H. Tan, and C. Jagadish, IEEE Photonics Technol. Lett. 18, 1648 (2006).

${ }^{13}$ T. M. Cockerill, D. V. Forbes, H. Han, B. A. Turkot, J. A. Dantzig, I. M. Robertson, and J. J. Coleman, J. Electron. Mater. 23, 115 (1994).

${ }^{14}$ M. Buda, J. Hay, H. H. Tan, J. Wong-Leung, and C. Jagadish, IEEE J. Quantum Electron. 39, 625 (2003).

${ }^{15}$ P. D. Siverns, S. Malik, G. McPherson, D. Childs, C. Roberts, R. Murray, B. A. Joyce, and H. Davock, Phys. Rev. B 58, R10127 (1998).

${ }^{16}$ T. Walther, A. G. Cullis, D. J. Norris, and M. Hopkinson, Phys. Rev. Lett. 86, 2381 (2001).

${ }^{17}$ P. Lever, M. Buda, H. H. Tan, and C. Jagadish, IEEE J. Quantum Electron. 40, 1410 (2004).

${ }^{18}$ K. Sears, Ph.D. thesis, The Australian National University, 2006. 